\title{
Three-Dimensional Ultrahigh-Resolution Optical Coherence Tomography of Macular Diseases
}

\author{
Ursula Schmidt-Erfurth, ${ }^{1}$ Rainer A. Leitgeb, ${ }^{2}$ Stephan Michels, ${ }^{1}$ Boris Považay, ${ }^{2}$ \\ Stefan Sacu, ${ }^{1}$ Boris Hermann, ${ }^{2}$ Christian Ablers, ${ }^{1}$ Harald Sattmann, ${ }^{2}$ Christopb Scholda, ${ }^{1}$ \\ Adolf F. Fercher, ${ }^{2}$ and Wolfgang Drexler ${ }^{2}$
}

Purpose. To demonstrate a new generation of three-dimensional (3-D) ultrahigh-resolution optical coherence tomography (UHR OCT) technology for visualization of macular diseases.

Methods. One hundred forty eyes with a distinct disease in each of the posterior pole compartments were examined with 3-D UHR OCT. 3-D imaging was performed with a high axial resolution of $3 \mu \mathrm{m}$ with a compact, commercially available, ultra-broad-bandwidth $(160 \mathrm{~nm})$ titanium:sapphire laser at a video rate of up to $25 \mathrm{~B}$-scans/s. Each tomogram consisted of $1024 \times 1024$ pixels, resulting in 25 megavoxels/s.

Results. 3-D UHR OCT offers high-precision 3-D visualization of macular diseases at all structural levels. The UHR modality allows identification of the contour of the hyaloid membrane, tractive forces of epiretinal membranes, and changes within the inner limiting membrane. The system provides quality 3-D images of the topographic dynamics of traction lines from the retinal surface down to the level of the photoreceptor segments. Intraretinal diseases are identified by their specific location in different layers of the neurosensory ultrastructure. Photoreceptor inner and outer segments are clearly delineated in configuration and size, with a characteristic peak in the subfoveal area. The microarchitecture of choroidal neovascularization is distinctly imaged, related leakage can be identified, and the volume can be quantified.

Conclusions. High-speed UHR OCT offers unprecedented, realistic, 3-D imaging of ocular diseases at all epi-, intra- and subretinal levels. A complete 3-D data set of the macular layers allows a comprehensive analysis of focal and diffuse diseases, as well as identification of dynamic pathomechanisms. (Invest Ophthalmol Vis Sci. 2005;46:3393-3402) DOI:10.1167/iovs.050370

From the ${ }^{1}$ Department of Ophthalmology, General Hospital of Vienna, Medical University of Vienna, Vienna, Austria; the ${ }^{2}$ Center for Biomedical Engineering and Physics, Christian Doppler Laboratory, Medical University of Vienna, Vienna, Austria.

Supported by Austrian Science Fund Grants P14218-PSY and Y159-PAT; the Christian Doppler Society; Femtolaser Produktions, GmbH (Vienna, Austria); and Carl Zeiss Meditec, Inc.

Submitted for publication March 23, 2005; revised April 26, May 20, May 26, 2005; accepted July 21, 2005.

Disclosure: U. Schmidt-Erfurth, None; R.A. Leitgeb, None; S. Michels, None; B. Považay, None; S. Sacu, None; B. Hermann, None; C. Ahlers, None; H. Sattmann, None; C. Scholda, None; A.F. Fercher, None; w. Drexler, Carl Zeiss Meditec, Inc. (C)

The publication costs of this article were defrayed in part by page charge payment. This article must therefore be marked advertisement in accordance with 18 U.S.C. $\$ 1734$ solely to indicate this fact.

Corresponding author: Wolfgang Drexler, Center for Biomedical Engineering and Physics, Christian Doppler Laboratory, Medical University of Vienna, Waehringer Strasse 13, A-1090 Vienna, Austria; wolfgang.drexler@meduniwien.ac.at.
$\mathrm{U}$ ltra-high-resolution optical coherence tomography (UHR OCT $)^{1-5}$ is a recently developed strategy that improves established OCT technology ${ }^{6-8}$ and achieves unprecedented in vivo visualization of subcellular features ${ }^{1}$ and retinal disease. ${ }^{2-5}$ UHR OCT exceeds standard-resolution OCT by obtaining superior axial image resolution of $3 \mu \mathrm{m}$ compared with the 10 - to $15-\mu \mathrm{m}$ threshold achieved so far in conventional retinal imaging and therefore offers enhanced visualization of all intraretinal layers. Improved resolution and acquisition speed allow, within 4 to 6 seconds, the performance of a noninvasive optical biopsy of the human retina-that is, visualization of the intraretinal morphology approaching a level of structural differentiation obtainable only with histopathology. This quantum leap in imaging performance is achieved by using a state of the art ultra-broad-bandwidth light source instead of conventional superluminescent diodes.

To evaluate the potential of UHR OCT for enhanced visualization of intraretinal structures and to provide a distinct base for correct interpretation of in vivo ophthalmic UHR OCT tomograms, preclinical studies have been conducted to correlate UHR OCT cross-sectional images of in vivo porcines and monkey ${ }^{10}$ (Macaca fascicularis) retinas with histologic sections of the identical specimen. The results of these comparative studies allow extraction of structural information from in vivo UHR ophthalmic OCT tomograms and substantially reduce the ambiguities in data interpretation. Analogies with histologic features allow correlation of about 10 distinct intraretinal layers with corresponding signals in UHR OCT tomograms. Recently, this novel OCT technology has been used in a clinical setting for the first time, enabling in vivo crosssectional imaging of macular diseases with an unprecedented axial resolution of $\sim 3 \mu \mathrm{m}$, giving the technology unique value in clinical imaging. ${ }^{4,11-13}$

In clinical practice, though, to detect early stage disease, to follow disease progression, and to monitor therapy-induced changes, it is best not to rely on a few B-scans (tomograms) randomly taken at sites that are presumptively chosen during image acquisition. An imaging modality, accomplishing UHR visualization of all intraretinal layers within a few seconds across a volume large enough to include the entire dimension of the investigated disease would clearly have a significant clinical impact on retinal diagnosis. With such a comprehensive 3-D technique, the decision regarding a specific imaging site would not have to be made during the process of data acquisition. Instead, the complete 3-D data volume could ideally be analyzed in more detail and at various locations after image processing, similar to the procedures used in computed tomography or magnetic resonance imaging.

First-generation, UHR OCT systems perform visualization of the tissue microstructure in the so-called time domain. Depth information in this case is obtained as a function of distance or time. State of the art delay lines have been developed to provide high scanning speeds of up to $8 \mathrm{kHz}$ (i.e., 8000 A-scans 
per second ${ }^{14}$ ), with the drawback of lower system sensitivity for faster scanning speed and/or broader optical bandwidth to accomplish UHR and longer depth range. A potential alternative to time domain OCT for high-speed imaging is a technique in which the entire spatially resolved tissue reflectance (Ascan) is obtained simultaneously, and no depth scanning is necessary. ${ }^{15-18}$ This technique has been referred to in the literature as Fourier domain OCT, spectral domain OCT, frequency domain OCT, and swept source OCT. The technical improvement can be accomplished either by dispersing the interferometric information in space by using a spectrometer setup (also referred to as Fourier domain OCT or spectral domain OCT in the literature) or by encoding information in time by tuning a monochromatic light source in combination with a single photodetector (also referred to in the literature as frequency domain OCT or swept source OCT). The acquisition speed for both approaches- using a spectrometer as a detector or a tuneable light source-is then mainly limited by the read-out rate of the charge-coupled device (CCD) camera or the tuning speed of the light source, respectively. Because of the decoupling of scanning range and electronic detection band width, both approaches offer a significant sensitivity advantage, allowing a dramatic increase in line rate (A-scan rate) without loosing imaging performance in comparison to time domain OCT. ${ }^{19-21}$ Accordingly, OCT with a spectrometer used for detection has recently been demonstrated to offer high speed, ${ }^{22-24} \mathrm{UHR}^{25-27}$ and functional imaging. ${ }^{28,29}$

One possible approach to performing 3-D retinal imaging is an extension of time domain OCT called en face OCT. ${ }^{30-34}$ This technique combines the transverse scanning approach of a confocal scanning laser ophthalmoscope (cSLO) with the depth-sectioning capabilities of OCT. This technique represents a cSLO with improved axial resolution by more than one order of magnitude, allowing high transverse resolution en face (also called C-mode scan) imaging. So far, 64 planes in depth, each consisting of $256 \times 128$ pixels, enabling approximately 2 million voxels (3-D data points)/second, resulting in limited cross-sectional (B-scan) visualization capability and a pixelized tomogram appearance.

In this pilot study, we present the first clinical evaluation of a second generation UHR OCT system with 3-D UHR OCT (3-D UHR OCT), based on Fourier domain OCT, providing video imaging of retinal diseases of all compartments (epiretinal, intra-, subretinal) of the posterior pole of the eye.

\section{Methods}

\section{3-D UHR OCT Technology}

3-D retinal imaging was performed with an axial resolution of $3 \mu \mathrm{m}$ with a compact, commercially available ultra-broad-bandwidth (160 $\mathrm{nm}$ ) titanium:sapphire laser (Integral; Femtolaser Produktions GmbH, Vienna, Austria) $)^{35}$ at a video rate of up to 25 B-scans/s. Each tomogram consisted of $1024 \times 1024$ pixels, resulting in 25 million voxels/s, more than one order of magnitude better than existing en face OCT techniques. Similar in vivo OCT sensitivity performance and visualization of intraretinal layers, especially the inner (ISPR) and outer segment (OSPR) of the photoreceptor layer, was achieved by 3-D UHR OCT compared with standard UHR OCT, with a 100-fold higher data-acquisition speed of 3-D UHR OCT, enabling unprecedented in vivo 3-D ultrahigh resolution imaging of retinal diseases.

For the present study, the retinal exposure had to account for the ultra-broad-bandwidth light generated by the laser. The ANSI standards $^{36}$ for retinal exposure account for wavelength, exposure duration, and multiple exposures of the same spot of the retina. Because the laser source generates femtosecond pulses, the laser output was coupled into a 100-m-long optic fiber that was used to provide dispersive stretching of the pulse duration to hundreds of picoseconds. This technique reduces the peak pulse intensities by several orders of magnitude and, because the laser operates at an $80-\mathrm{MHz}$ repetition rate, the output can be handled as a continuous wave. OCT imaging was performed with axial scans at rates ranging from 10,000 to 25,000 $\mathrm{Hz}$, resulting in a retinal exposure time at each point where an axial scan of 40 to $100 \mu$ s was acquired. OCT imaging was performed with $800-\mu \mathrm{W}$ incident optical power in the scanning OCT beam, which is well below the ANSI exposure limits. Each volume, acquired in 2.5 to 6 seconds (depending on the A-scan rate, which was chosen according to the quality of the ocular media of the patients), consisted of 60 tomograms, each comprising 1024 A-scans moving in a transverse direction with 1024 axial pixels, covering a diseased area of approximately $3 \times 3 \mathrm{~mm}$ (except for the disease shown in Fig. 5, where $3 \times$ $5 \mathrm{~mm}$ was scanned and in Figs. $6 \mathrm{~A}-\mathrm{E}$, where $2 \times 3 \mathrm{~mm}$ was scanned), at a depth of approximately $1 \mathrm{~mm}$. With this incident power, a sensitivity of $95 \mathrm{~dB}$ was achieved for all scanning conditions. The sensitivity of the presented technique was measured with an artificial eye consisting of a mirror and $25 \mathrm{~mm}$ of water in combination with a neutral-density filter. With the same optical power and bandwidth as is used in clinical in vivo measurements, the density of the optical filter in front of the artificial eye was increased until a signal above the noise could still be distinguished. The maximum of this signal was then used in combination with the variance of the noise and the value of the optical neutral-density filter to calculate the best sensitivity of the system.

\section{Patient Selection and Imaging Procedure}

All investigations adhered to the tenets of the Declaration of Helsinki. Written informed consent was obtained from all individuals in the study after a detailed explanation of the nature and possible consequences of the study procedures. The study was approved by the Ethics Committee of the Medical University Vienna, School of Medicine. All 3-D ultrahigh resolution OCT data in the present study were acquired with $\sim 3-\mu \mathrm{m}$ axial and approximately 15 - to $20-\mu \mathrm{m}$ transverse resolution

One hundred forty eyes with distinct macular diseases of different origins and locations were included. The primary diagnosis was based on a thorough clinical examination that included visual acuity testing, slit lamp examination, and ophthalmoscopy. In cases demonstrating an exudative component clinically, fluorescein angiography (FA) was added to the diagnostic procedure. Eyes were grouped into diseases with a primary epiretinal location including epiretinal membranes and vitreomacular traction, with or without macular hole; an intraretinal disease such as cystoid macular edema, central serous chorioretinopathy, hereditary retinal disease; or an a priori subretinal location, such as age-related macular degeneration with choroidal neovascularization $(\mathrm{CNV})$, dry changes such as drusen or atrophy of the retinal pigment epithelium (RPE), or a serous detachment of the pigment epithelium (PED).

\section{Results}

\section{3-D UHR OCT Imaging of the Physiologic Macular Configuration}

The retina within the macular region demonstrates a characteristic 3-D appearance in 3-D UHR OCT imaging. Each 3-D image consists currently of a series of 60 tomograms, each 3 $\mathrm{mm}$ in horizontal length, taken over a vertical distance of $3 \mathrm{~mm}$ and an axial depth of $1 \mathrm{~mm}$, resulting in a $3 \times 3 \times 1 \mathrm{~mm}$ optical specimen. The serial presentation offers a comprehensive 3-D view of the macular architecture (Fig. 1). Using a video-mode movie technique, the specimen can be rotated around its axis and can be viewed from different perspectives. The view from the 6 o'clock position centered on the first tomographic section at the specimen margin inferior to the fovea demonstrates the contour of the retinal surface on top delineated by the hyperreflectivity of the inner limiting membrane (ILM) and the 

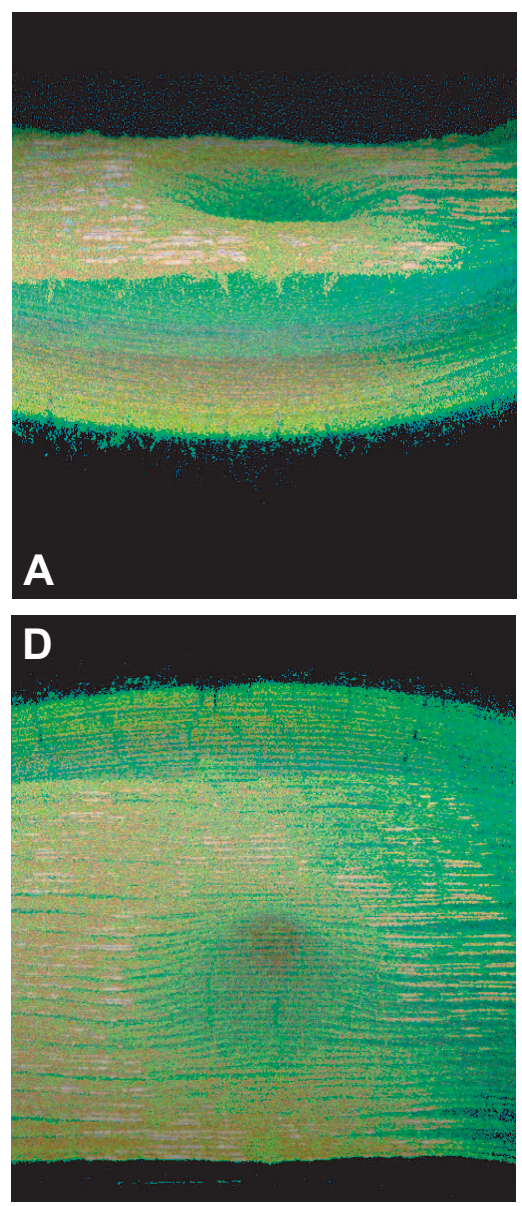
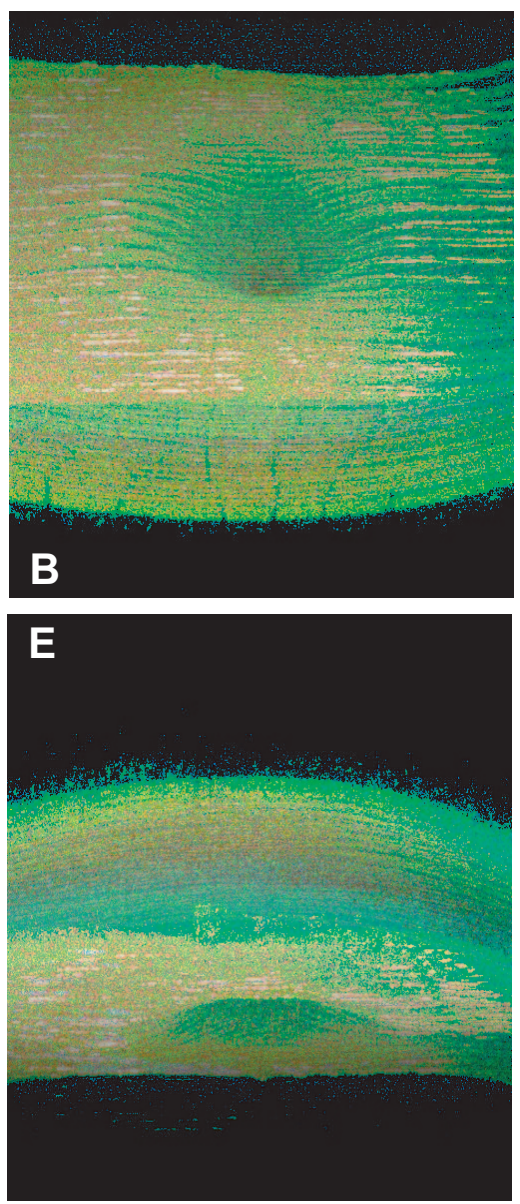
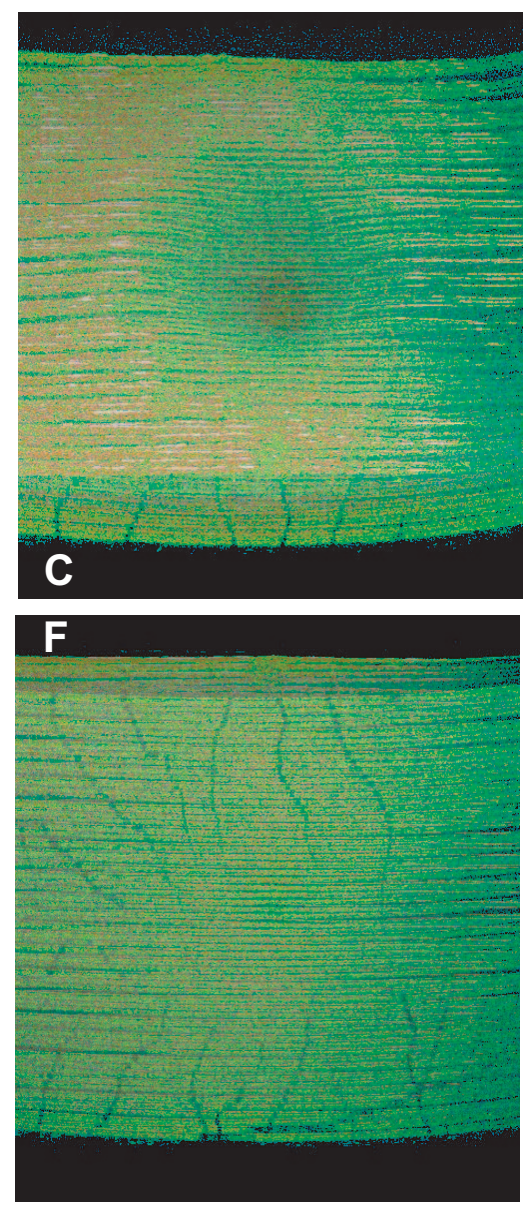

FIGURE 1. 3-D UHR OCT imaging of the physiologic macula (Movies 1, 2, http://www.iovs.org/cgi/content/full/46/9/3393/DC1). Different views of a 3-D UHR OCT representation of a normal human fovea are depicted. The 3-D representation is constructed from of 60 tomograms, each consisting of 1024 A-scans with 1024 sample points, covering an area of approximately $3 \times 3 \times 1 \mathrm{~mm}$, acquired in approximately 4 to 5 seconds. Comprehensive visualization of the topography and morphology of the entire foveal depression (A-E) and the pattern of the retinal vascular net (F), consistent with an angiographic image, is demonstrated.

nerve fiber layer (NFL) and the convex contour of the hyperreflective $\mathrm{RPE} / \mathrm{ch}$ oroid band on the bottom. The retina at this location appears relatively thick at a distance of approximately $1.5 \mathrm{~mm}$ apart from the foveal center (Fig. 1A). The retinal surface in the subsequent section located behind this first section is even more prominent, due to the radial distribution of the NFL in the area of the concentric macular ring reflex. A more transverse view of the 3-D UHR OCT specimen offers a comprehensive view of the topography and morphology of the entire foveal depression. The ILM/NFL bands of all 60 sections form a smooth concavity in the center of the 3-D macular specimen (Fig. 1B). Accordingly, a front view of the macular surface produces an almost flat image of the macula, with a dark round area in the center corresponding to the fovea (Fig. 1C). When the optical sample is further rotated during video animation, the foveal depression is highlighted from the 12 o'clock perspective, superior to the fovea (Fig. 1D), which completes the 3-D evaluation of a regular foveal contour in all perspectives. The direct view on the last and uppermost tomographic section represents an upside-down perspective of the macular specimen with the convex contour of the hyperreflective RPE-choroid band on top and the concave surface of the ILM-NFL band on the bottom (Fig. 1E).

The direct view on the posterior pole of the RPE-choroid curvature exhibits a comprehensive pattern of the retinal vascular net consistent with an angiographic image, but without the use of a fluorescent marker. The visualization of the vasculature is entirely due to the detection of the reflectance shadows from the overlying vascular walls (Fig. 1F).

\section{Analysis of Single UHR OCT Scans from Serial Sections}

Figure 2A demonstrates an OCT view of the fundus similar to the one obtained by standard fundus photography, which can be directly reproduced from 3-D UHR OCT data. This OCT view is generated by summing the A-scan signal along the axial direction, resulting in a brightness pixel value for each axial scan, and can be used to register directly the UHR OCT tomograms (Figs. 2C-E), in addition to a 3-D representation of the imaged volume (Fig. 2B). Since each scan of the 60 sections consists of 1024 A-scans with 1024 data points, a single tomographic scan can be extracted and analyzed separately to identify a specific site of interest. Even the architecture of the normal macula varies substantially, depending on the precise location and the distance from the foveal center. When tomographic images from the extrafoveal area, taken at a distance of $1 \mathrm{~mm}$ from the foveal center, are selected, the retinal band is homogenous and wide, and no central thinning is seen (Fig. 2C). However, in the depth of the ganglion cell layer (GCL) and inner plexiform layer (IPL) below the ILM/NFL, abundant areas of focal hyperreflectivity are visible that originate from large- 

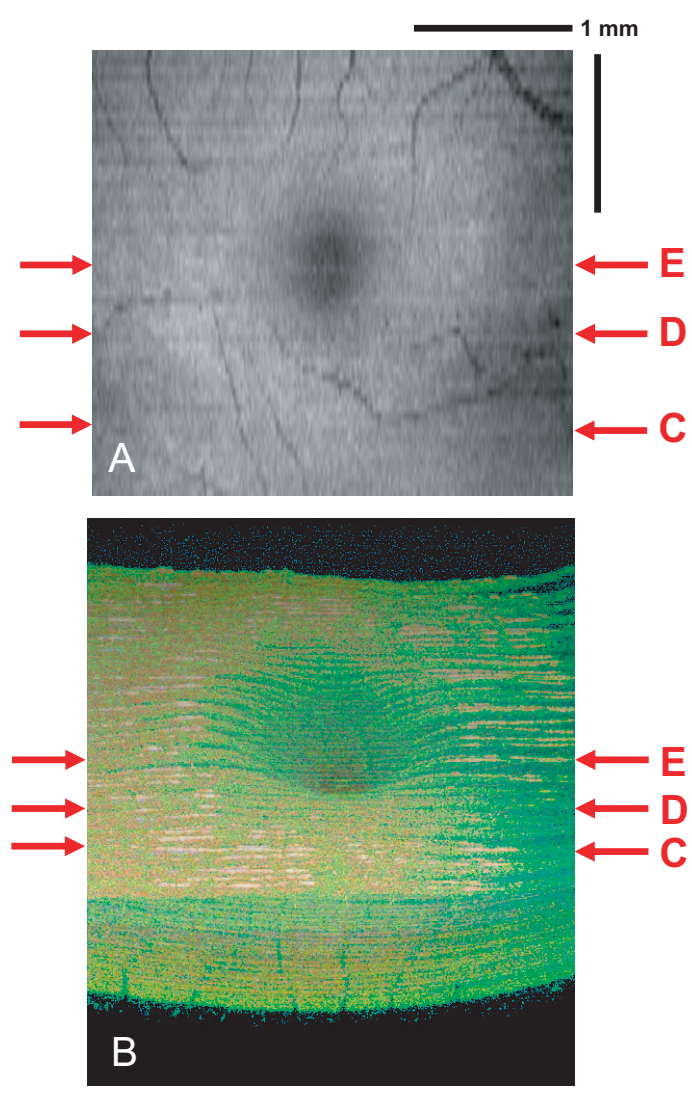
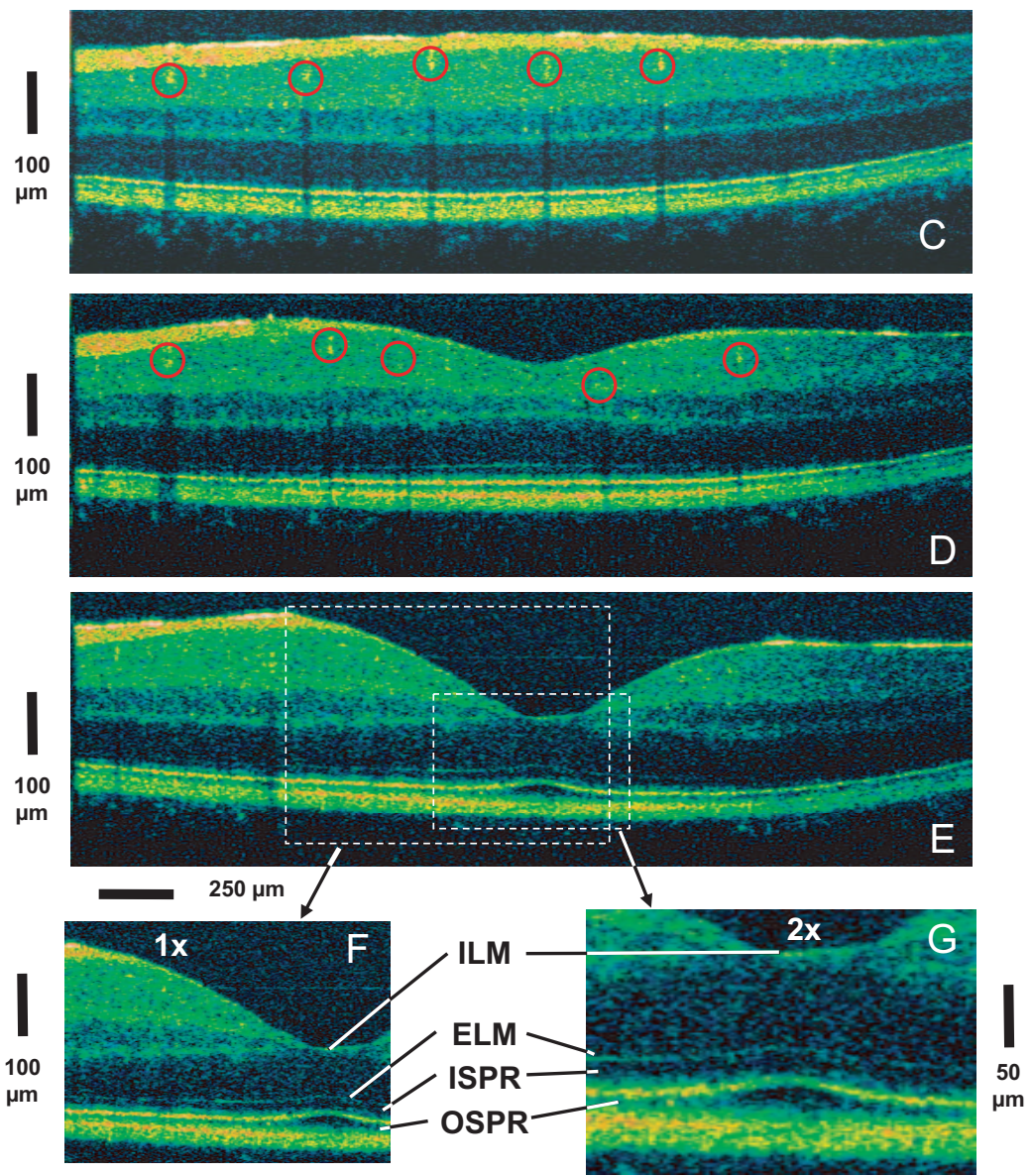

FigURE 2. Single UHR OCT tomograms from the 3-D UHR OCT data set (Movies 1, 2, http://www.iovs.org/cgi/content/full/46/9/3393/DC1). OCT fundus view, generated from the UHR OCT A-scans (A) as well as 3-D UHR OCT overview $(\mathbf{B} ; 3 \times 3 \times 1 \mathrm{~mm})$ indicate the exact corresponding location of the tomograms depicted in (C-E). Focal hyporeflectivity that originates from large-diameter vessels such as arterioles and venules $(\mathbf{C}, \mathbf{D}$, red circles) and a clear increase of outer cone segments in the central foveal region are depicted, in addition to all other major intraretinal layers (E-G). Rectangles: magnified views depicted in (F) and (G).

diameter vessels such as arterioles and venules located on the surface of the retina. The solid vascular walls produce distinct zones of reflectance shadows throughout the underlying layers (cf. Figs. 2C, 2D, red circles). Otherwise, the 10-layered structure of the retina appears as detailed as seen in conventional two-dimensional (2-D) UHR OCT without any loss of axial resolution. Approaching the juxtafoveal area, the center of the tomogram section becomes thinner with the attenuation of the nerve fiber density (Fig. 2D). Smaller-caliber blood vessels are located deeper in the retina (i.e., within the inner nuclear layer [INL]) and appear less hyperreflective with a narrower shadow. The tomographic sections from the center of the tomographic series, clearly delineate the central foveal depression (Fig. 2E). Retinal vessels and associated hyporeflective shadows are completely absent. Characteristic features of the central foveal area are an opacification of the ILM at the deepest location (cf., Figs. 2F and 2G) of the depression, consistent with a solid structural contact zone between the posterior hyaloid membrane (not visualized), the ILM, and Müller cells (not visualized) on the surface of the fovea and a focal elevation of the external limiting membrane (ELM) and the junctional band between the ISPR and OSPR on the bottom of the foveal retina (cf. Figs. $2 \mathrm{~F}$ and $2 \mathrm{G}$ ). This prominence is due to the increased length of the subfoveal cone outer segments in the foveal center, compared with the relatively shorter OSPR in the extrafoveal retina.

\section{Diseases of the Vitreomacular Interface}

Diseases of the vitreomacular interface in the macular area may exert traction in vertical as well as in axial direction and lead to distortion of the retinal layers and loss of the foveal depression. The alteration of the retinal surface is easily identified in the appearance of the OCT fundus view (Fig. 3A) as well as in the 3-D overview of the UHR OCT tomography (Fig. 3B). Analysis of the single scans in selected locations adds substantial information regarding the distribution of the traction forces and the resulting effects on the intraretinal organization. The single scans clearly demonstrate the vertical direction of traction. A horizontal epiretinal membrane leads to a fine overall wrinkling of the ILM surface. It is not apparent from the 3-D overview image (Fig. 3B) that the thickened gliotic membrane is attached in the nasal portion (right side), but is detached from the temporal portion (left side; Figs. 3C, 3E) of the retina. The contour of the posterior hyaloid membrane (cf. Figs. 3C and $3 \mathrm{E}$, white arrows) is visible in the detached left portion as a continuation of the attached gliotic membrane on the right and indicates that the disease originates from shrinkage of the posterior hyaloidal membrane rather than from a separate epiretinal membrane formation. The layered retinal architecture is intact within the superficial portion of the retina, structural changes are distinctly located in the deep portion of the retina beyond the outer nuclear layer (ONL; cf. areas indicated by white arrows in Fig. 3D). In more superior scans, the 

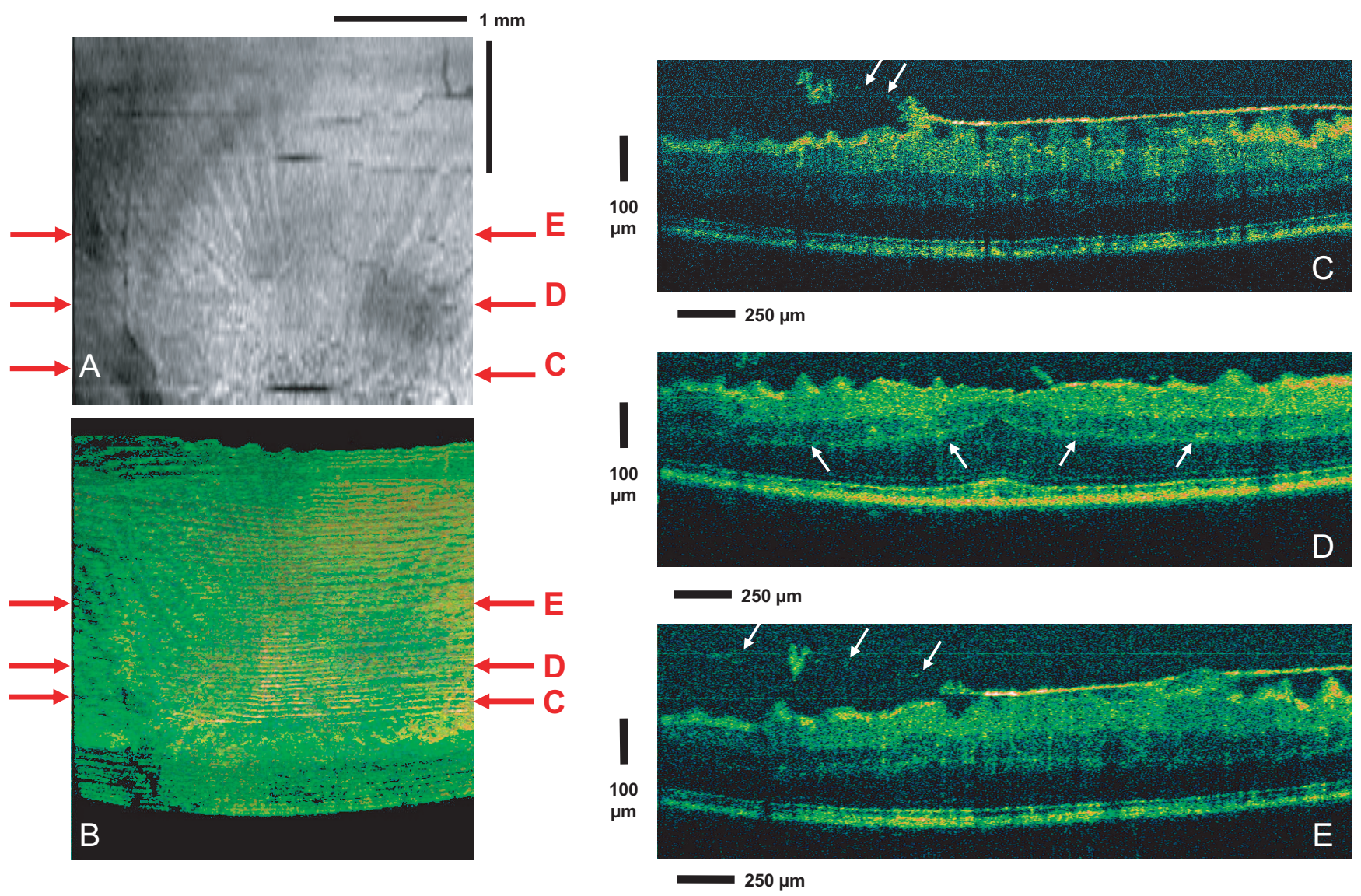

FiguRE 3. Diseases of the vitreomacular interface: detachment and shrinkage of the posterior hyaloid membrane (Movies, 3, 4, http://www.iovs. $\mathrm{org} / \mathrm{cgi} / \mathrm{content} / \mathrm{full} / 46 / 9 / 3393 / \mathrm{DC} 1)$. OCT fundus view generated from the UHR OCT A-scans $(\mathbf{A})$ and a 3 -D UHR OCT overview $(\mathbf{B} ; 3 \times 3 \times 1$ $\mathrm{mm}$ ) indicate the exact corresponding location of the tomograms depicted in (C-E). Clear visualization of overall wrinkling of the ILM surface and the thickened gliotic hyaloid membrane is obtained $(\mathbf{C}, \mathbf{D})$. The membrane is detached from the foveal center and no signs of an imminent hole formation are present (E). The posterior hyaloid membrane, indicated by two arrows, is shown in (C) and (E). (C-E) Distortion of the retinal architecture is visible. (D, arrows): the ONL.

epiretinal membrane is attached in a continuous connection and without wrinkling to the ILM and NFL without significant impact on the retinal layer integrity (Fig. 3D). The sensory retina in the entire section is markedly thickened due to vertical stretching. The foveal depression has disappeared completely, but the fovea can be identified by the characteristically prominent OSPR region (Figs. 3D, 3E). The membrane is detached from the foveal center and no signs of imminent hole formation are present.

\section{Intraretinal Diseases with Macular Hole}

Vitreomacular traction in an axial direction leads to a completely different appearance of the 3-D ultrastructure of the retina. Figure $4 \mathrm{~A}$ depicts the OCT fundus view and indicates the location of the extracted tomograms shown in Figures 4C-G. The 3-D overview illustrates a loss of the foveal contour and a distinct central elevation of the retinal surface (Fig. 4B). In the extrafoveal region, no traction is seen; however, cystic changes are located in the superior portion of the retina, mostly within the GCL and INL (Fig. 4C and twofold magnified view in Fig. 4D). In the juxtafoveal area the localized attachment of the posterior hyaloid membrane is clearly identified as well as the resultant distortion of the intraretinal layers throughout the entire thickness of the retina (Fig. 4E). The foveolar site of focal traction on the ILM leads to a rupture of the central retina with formation of a small, but full-thickness macular hole (Fig. 4F). Superior to the fovea and outside of the defect, the retina demonstrates massive cystic changes most intensively within the deeper portion of the retina, including the ONL and, to a lesser extent, within the superficial layers (Fig. 4G).

\section{Primary Disease of the RPE Layer}

3-D UHR imaging of an eye with bull's eye dystrophy demonstrates characteristic pathognomonic features. Figure $5 \mathrm{~A}$ depicts the OCT fundus view and indicates the location of the extracted tomograms shown in Figures 5E-I. The surface topography shows a round, irregular elevation of the perifoveal retinal surface and a loss of the foveal depression (Fig. 5B). The rotated side view of the optical specimen outlines significant alterations of the intraretinal structures as well as two bandlike extrusions of hyperreflectivity toward the choroid (Fig. 5C). Further rotation, imaging the specimen from the choroid-RPE side, shows that the hyperreflective bands form a characteristic ring of hyperreflectance in the choroid (Fig. 5D). A single UHR OCT scan (Fig. 5E) taken through the fovea demonstrates an elevation with loss of RPE hyperreflectance ( + phenomenon) consistent with an absence of RPE pigmentation clinically (cf. asterisks in Fig. 5F and images in Figs. 5C and 5D). Intraretinal changes include a thinning of the ONL and an absence of the ISPR and OSPR bands in areas of RPE loss (cf. Fig. 5F: Area of intraretinal layer impairment is indicated by the red rectangle). Superior to this site, two perifoveal areas of hyperreflectance within the RPE band are detectable. However, below these 

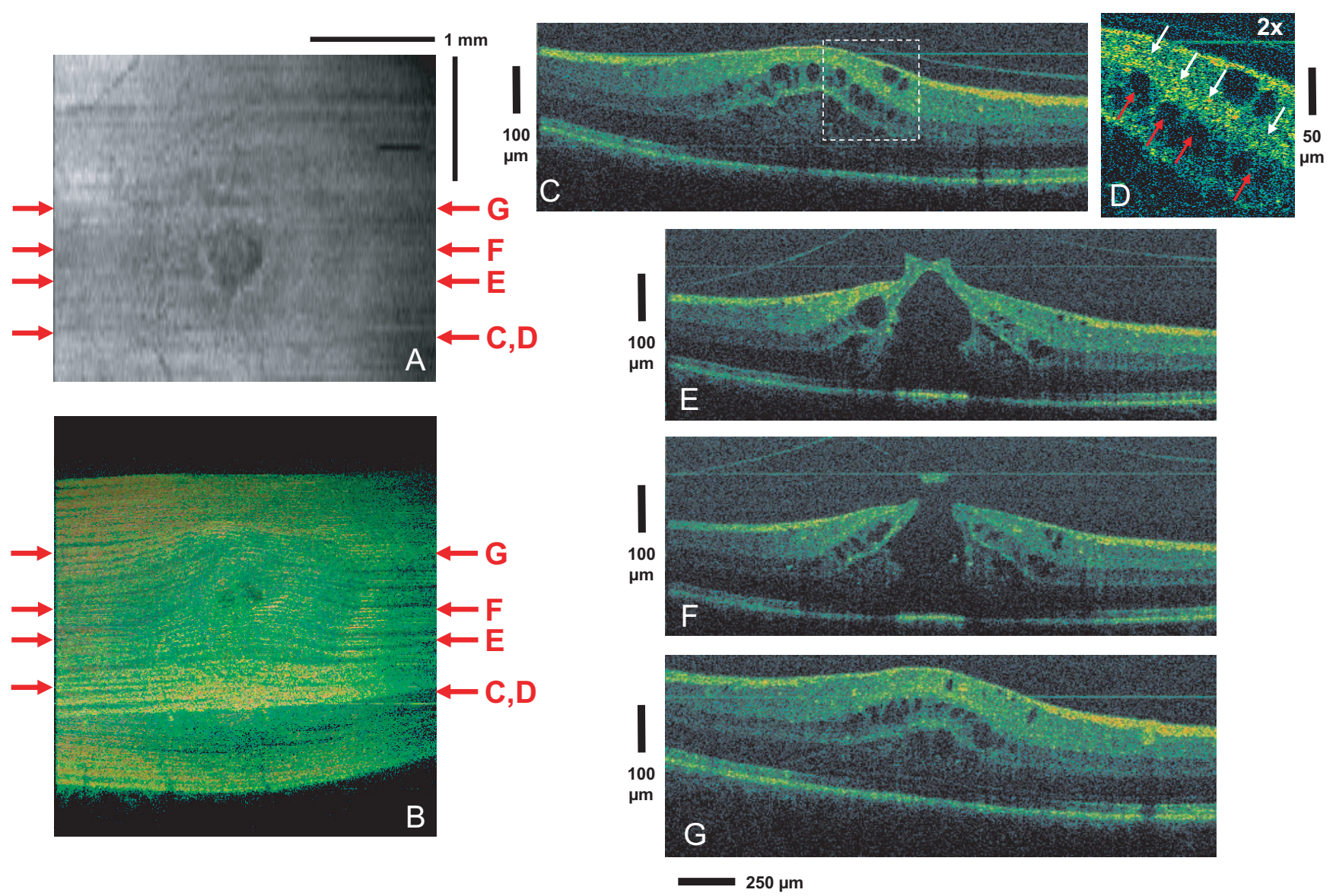

FIGURE 4. Different stages of intraretinal diseases with vitreomacular traction and macular hole formation (Movies 5, 6, http://www.iovs.org/ cgi/content/full/46/9/3393/DC1). OCT fundus view, generated from the UHR OCT A-scans (A) and a 3-D UHR OCT overview (B; $3 \times 3 \times 1 \mathrm{~mm})$ indicate the exact corresponding location of the tomograms depicted in (C-G). 3-D overview illustrates a loss of the foveal contour and a distinct central elevation of the retinal surface (B). In the extrafoveal region, no traction is visible; however, cystic changes are located in the GCL (C) and the INL (G). (D) Twofold magnification of the area in the rectangle in (C), with the IPL (white arrows) and INL (red arrows) indicated. In the juxtafoveal area, the localized attachment of the posterior hyaloid membrane is clearly identified as well as the resultant distortion of the intraretinal layers throughout the entire thickness of the retina (E) finally resulting in the disruption of retinal integrity and the formation of a hole (F).

areas, vertical bands of hyperreflectance allow better visualization of choroidal structures, indicating a loss of integrity of the RPE (Fig. 5G, and also 5C, 5D). Scans taken superior to the fovea demonstrate similar changes in the intraretinal architecture, including the loss of the junctional band between the ISPR and OSPR bands (cf. Fig. 5I, arrows) The two hyperreflective elevations on the RPE band become confluent centrally and intrachoroidal hyperreflective bands (e.g., asterisk in Fig. 5I) merge, creating the prominent choroidal ring pattern ( - phenomenon) within the inferior curvature of the ring structure visible in the rotated specimen (Figs. 5G, 5H, 5I and also 5C, 5D).

\section{Subretinal Disease with CNV}

Subretinal diseases in age-related macular degeneration can vary, even in the same patient, as documented in the following images. Figure 6A depicts the OCT fundus view, covering the area imaged by 3-D UHR OCT. There is a discrete central swelling of the foveal retina. FA demonstrates active extravasation from a subfoveal neovascular lesion (Fig. 6B). The locations of the 2-D scans shown in Figures 6C-E are indicated in the OCT fundus view (Fig. 6A). 3-D UHR OCT outlines an elevation of the central macular contour and single UHR scans taken through the fovea (Figs. 6C-E) show the changes associated with CNV. The foveal depression is reduced in depth, but the retinal architecture is completely preserved up to the ELM (Fig. 6C). Superior to the fovea, the focal detachment is filled with serous fluid (Fig. 6C). The fluid is located underneath the partially interrupted junctional band between the ISPRs and OSPRs, and the RPE band appears continuous. In the next image, a focal irregularity is identified at the level of the RPE and Bruch's membrane (Fig. 6D). The full extent of RPE and Bruch's membrane erosion is seen in the foveal scan, were the RPE band is absent in the center of the lesion and the neovascular mass has grown through the entrance site and has entered and filled the subretinal space (Fig. 6E).

The fellow eye (Figs. 6F-K) shows a central areolar atrophy, clearly outlined by the OCT fundus view (Fig. 6F) as well as indocyanine green angiography (ICGA; Fig. 6G). Corresponding locations of the scans shown in Figures $6 \mathrm{H}-\mathrm{K}$ are indicated in the OCT fundus view (Fig. 6F). 3-D UHR OCT shows a widened foveal depression of increased depth (Figs. 6H-K). Outside the area of RPE atrophy, the retinal architecture is completely preserved down to the photoreceptors and the RPE band. However, there is a sudden change in the retinal structure overlaying the RPE atrophy. The junctional band between the ISPRs and OSPRs is completely lost, as are the overlying retinal structures up to about the IPL (cf. Fig. 6K, red arrows indicating the intact band), a loss of the ELM (cf. Fig. 6K, white arrows indicating the intact ELM), as well as a hyperreflectance 


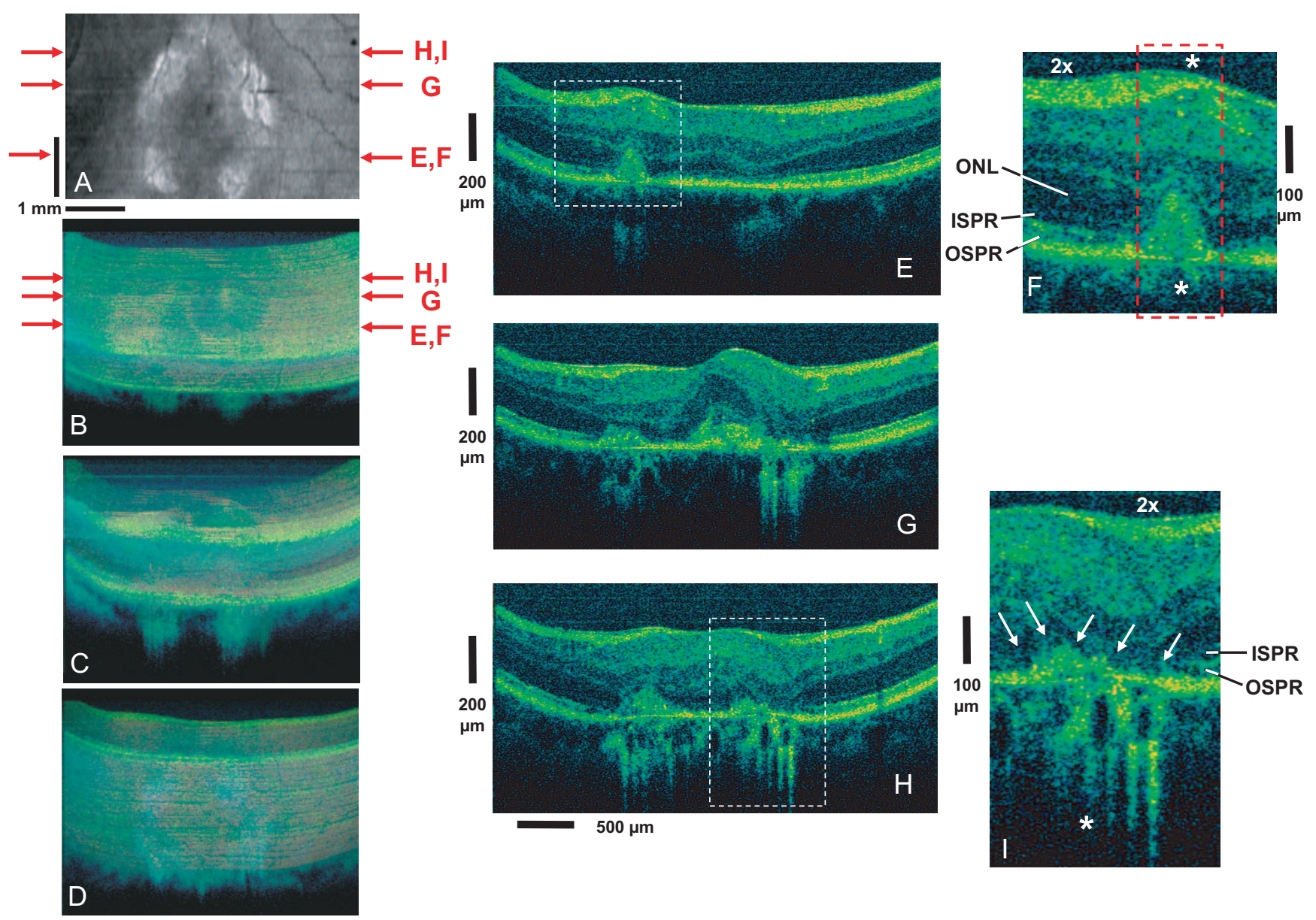

Figure 5. Primary disease of the RPE layer showing bull's eye dystrophy (Movies 7, 8, http://www.iovs.org/cgi/content/full/46/9/3393/DC1). OCT fundus view, generated from the UHR OCT A-scans (A) and a 3-D UHR OCT overview $(\mathrm{B} ; 3 \times 5 \times 1 \mathrm{~mm})$ indicate the exact corresponding location of the tomograms depicted in (E-I). Different views of a 3-D UHR OCT representation are shown in $(\mathbf{B})$ and $(\mathbf{C} ; 3 \times 5 \times 1 \mathrm{~mm})$. The surface topography shows a round, irregular elevation of the perifoveal retinal surface and the loss of foveal depression (B), two bands for hyperreflectivity within the choroid (C), and a characteristic ring of hyperreflectance in the choroidal overview (D). Single UHR OCT scans (E-I) taken through the fovea imply thickening of the inner retinal layers, appearing as an inverse traction to the RPE-choroid. Also shown are intraretinal changes such as thinning of the ONL and an absence of the ISPR and OSPR bands in areas of RPE loss (cf. F: area of intraretinal layer impairment is indicated by the red rectangle as well as arrows in I). The two hyperreflective elevations on the RPE band become confluent centrally and intrachoroidal hyperreflective bands (e.g., I, *) merged, creating a prominent choroidal ring pattern within the inferior curvature of the ring structure seen in the rotated specimen $(\mathbf{G}-\mathbf{I}$ and also $\mathbf{C}, \mathbf{D})$. (E, H, rectangles) Areas shown at higher magnification in $(\mathbf{F})$ and $(\mathbf{I})$.

window toward the choroids. Only proximal layers (e.g., the GCL) seem relatively well preserved. Due to complete loss of the RPE, consistent with a window defect, UHR OCT imaging reaches deeper into the choroid because there is less absorption and scattering at the melanin-rich RPE layer.

\section{Discussion}

A novel technology, 3-D ultrahigh resolution OCT, is presented for the first time with its unique clinical potential. The technique allows the precise delineation of the surface configuration of the macula (e.g., with thickening due to edema or proliferative disease) and distortions of the retinal surface due to vitreomacular traction or a loss in retinal substance in areas of atrophy. 3-D imaging is more detailed than stereoscopic ophthalmoscopy or photography, as it may be readily documented and quantified reproducibly. Animated video techniques allow the rotation of the optical sample so that selected features may be identified more clearly. The varying perspectives allow the clinician to obtain a transparent "glass model" of the retina with the pathologic changes highlighted by remodeling their contour and consistency. Based on such a compre- hensive spatial overview, the dimension and volume of a disease may be measured and quantified in square millimeters. The volumetric measurement offers realistic dimensions, because the 3-D sample is imaged by 60 or more parallel sections and not extrapolated from six radial cuts only, with most of the tissue area not covered by the imaging scan, which is the technique used in conventional OCT mapping. ${ }^{7,8}$ Although 60 B-scans across an area of $3 \times 3$ or $3 \times 5 \mathrm{~mm}$ have been used in the present study, resulting in anisotropic 3-D sampling, it was our intention to demonstrate that motion artifacts within a B-scan are more significantly reduced with this technique. Our second goal was to demonstrate that raster scanning of a whole retinal volume- even in an anisotropic fashion-helps the clinician to avoid missing any important retinal features in the investigated area compared with effectiveness in that regard of the state of the art, commercially available system. Further developments in data acquisition technology will allow 3-D isotropic sampling of retinal volumes in the future.

In addition, the dynamic forces and distribution of distinct diseases are monitored closely with reconstruction of traction forces and identification of small, localized changes such as the disruption of an epiretinal membrane, the focal break in the 

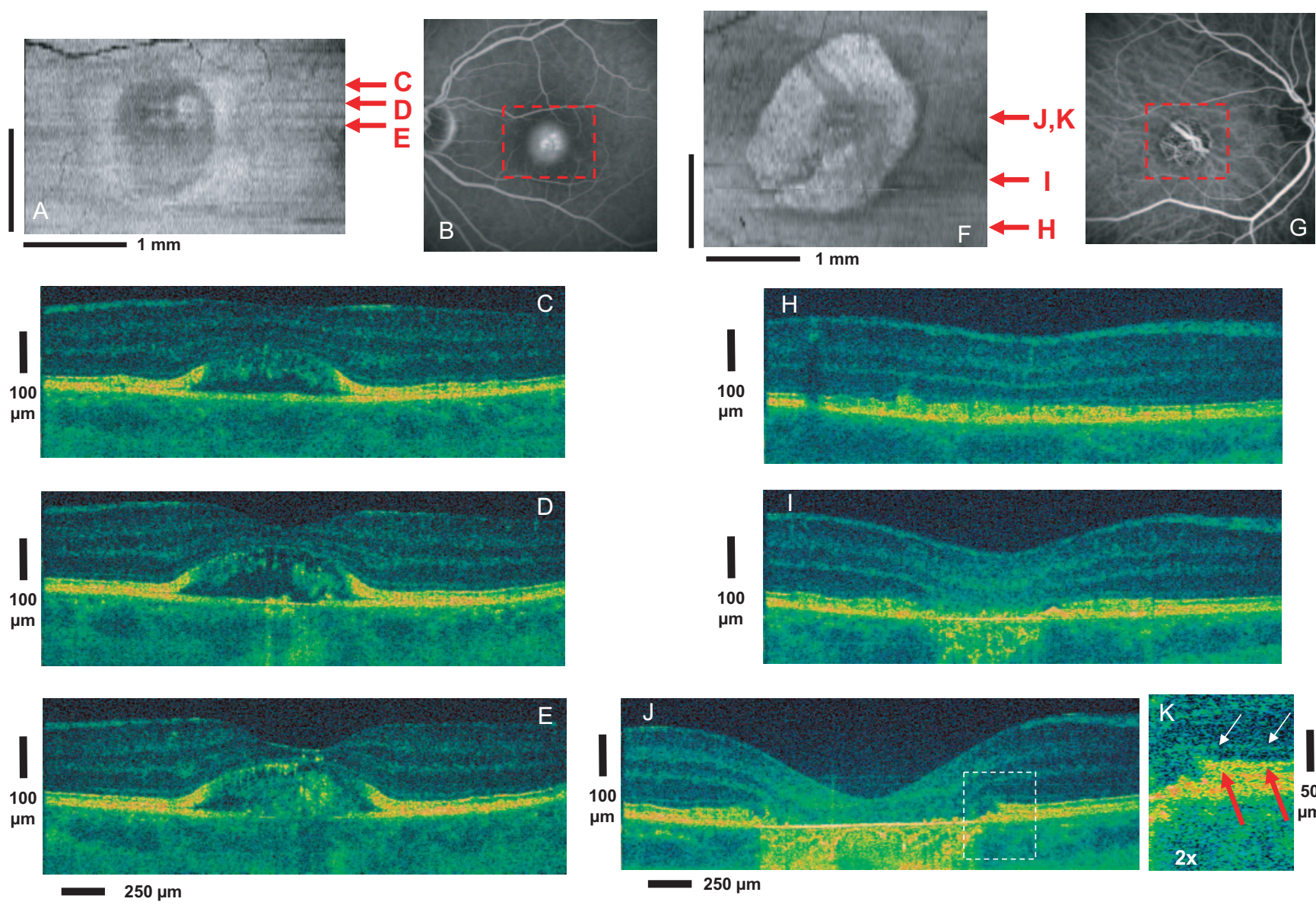

Figure 6. Subretinal disease with CNV. OCT fundus view, covering the area imaged by 3-D UHR OCT (A). FA illustrates the exudative activity of the neovascular lesion (B). Corresponding locations of the scans shown in (C-E) are indicated in (A). 2-D UHR OCT tomograms (C-E) outline an elevation of the central macular contour and show the changes associated with classic CNV. The CNV is located under the central partially interrupted junctional band between the ISPR and the OSPR bands. Large parts of the CNV are above the RPE band, which is partially disrupted (Movies 9, 10, http://www.iovs.org/cgi/content/full/46/9/3393/DC1: Please note that in 3-D Movie 9, the serous fluid of the CNV is visible to the viewer in red in the retina). The fellow eye shows central areolar atrophy: OCT fundus view (F), ICGA (G) indicating (red square) scanned area. Single scans illustrate sections with regular macular architecture $(\mathbf{H})$, and the transitional zone with RPE attenuation and loss of retinal integrity (I). The central UHR OCT scan (J) shows a widened foveal depression of increased depth, a complete loss of the ISPR and OSPR bands (cf. K, red arrows: intact band), a loss of the ELM (cf. $\mathbf{K}$, white arrows: intact ELM), as well as a hyperreflectance window toward the choroid. (J, rectangle) Area shown at higher magnification in (K) (Movies 11, 12, http://www.iovs.org/cgi/content/full/46/9/3393/DC1: Both movies do not display the whole retinal volume acquired, due to a strong motion artifact of the patient toward the end of the scan. This motion artifact does not affect the OCT fundus image depicted in $\mathbf{F}$ ).

ILM membrane and hole operculum, or the penetration site of the CNV through Bruch's membrane. 3-D angiography is another 3-D imaging method that provides a realistic representation of 3-D structural changes; however, it is restricted to the identification of vascular structures only. ${ }^{37}$ The retinal thickness analyzer (RTA) is limited to the detection of a surface contour, the epiretinal ILM layer, and a deep contour, the subretinal RPE layer, and does not provide any information about structural changes between these two borders.

The second breakthrough in quality is the high 2-D resolution of the UHR technique which, after the comprehensive 3-D representation, allows a detailed histologic sectioning of each 3-D site of interest. This feature permits insight into the intraretinal consequences of epiretinal traction from cystic changes within the superficial layers down to misalignment of the photoreceptor segments. A detailed scan-by-scan analysis after data acquisition reveals undetected focal changes and details of histologic changes, such as characteristics of macular hole formation or neovascular growth. Diseases can be located in relation to the intraretinal layer, and the primary site of damage can be identified and is correlated to the overall 3-D feature. Most important, the functional consequences of macular disease may be identified, with a precise representation of the photoreceptor integrity and location of the foveola. Small and early changes or changes outside the fovea do not escape the imaging process. Details such as the morphologic differences between cysts with pseudohole formation and lamellar macular holes are of substantial clinical relevance, and conventional OCT allows only a rough differentiation between both entities. ${ }^{38}$ Early stages of incomplete hole formation can easily be detected in fellow eyes. Such formations may not be imaged by conventional OCT, which is a major diagnostic problem. The accidental orientation and location of the scan set by the examiner has no influence on image quality or detection rate of small lesions, as all sections may be screened retrospectively and, with the high resolution used, any disease within the $3 \times$ 3 - or $3 \times 5-\mathrm{mm}$ field is identified. A precise quantification of lesions is also provided, such as the diameter and configuration of a macular hole which is an important prognostic factor for surgical intervention and influences the technique selected by the surgeon. ${ }^{39}$ Chan et al. ${ }^{40}$ found a so-called stage 0 macular hole in $28 \%$ of fellow eyes using advanced conventional OCT 
techniques. These stage 0 changes are defined as the presence of a preretinal, minimally reflective, thin band inserting obliquely on at least one side of the fovea. ${ }^{40}$ The topography of such changes is not recognized by 2-D OCT alone, but is identified with all its dynamics by 3-D UHR OCT. The improved resolution of 2-D UHR OCT enables imaging of previously unidentified changes in the photoreceptor morphology associated with macular hole and postoperative repair. ${ }^{11} 3$-D UHR OCT allows identification of isolated or multiple focal changes as well as their specific distribution throughout the macular area. Visualization of the junction between the ISPR and OSPR was shown to be an important indicator of photoreceptor integrity. ${ }^{11}$ The level of photoreceptor disturbance clearly determines the functional prognosis of any therapeutic intervention, which becomes a major issue with emerging pharmacologic interventions that have unknown end points of treatment and carry significant costs. High-resolution 3-D OCT is the appropriate noninvasive and precise modality to document the value of selective modern strategies where conventional techniques such as stereoscopic ophthalmoscopy and angiography fail to identify ultrastructural changes and the mechanisms of the therapeutical strategy. Novel pharmacologic modalities (e.g., intravitreal steroids or antiangiogenic agents) avoid mechanical manipulation associated with earlier techniques, such as surgery, laser coagulation, or photodynamic therapy, and work by mechanisms that are neither completely understood nor identified in their tissue interaction. Adequate diagnostic modalities combining a solid stereoscopic evaluation of the treatment effect and a detailed focal resolution of the ultrastructural mechanisms are urgently needed. 3-D UHR OCT characteristics perfectly fulfill this requirement and will allow the clinical development of these new strategies to proceed.

3-D UHR OCT enables unprecedented visualization of all major intraretinal layers, which had previously been possible only with histopathology. Clear visualization of all the major intraretinal layers and the ability to assess changes of retinal morphology associated with retinal diseases in an early stage, especially in the ISPR-OSPR/ELM/RPE complex, promises to have a significant impact on diagnosis and investigation and to provide a better understanding of pathogenesis and evaluation of novel therapy approaches for a variety of macular diseases. The hypothesis is that intraretinal structures that are relevant for the diagnosis and monitoring of early stages of eye diseases can be resolved by the proposed optical biopsy version of 3-D UHR OCT.

It is unlikely that OCT will replace histology or other existing ophthalmic diagnostic modalities. However, from the viewpoint of screening and diagnosis of diseases, the proposed version of OCT may enable significantly new insight into the pathogenesis and therapeutic control of several retinal diseases. The unique features of this developed technology would enable a broad range of research and clinical applications, which may not only complement many of the existing ophthalmic imaging technologies available today, but also may reveal previously unseen intraretinal changes.

This quantum leap in imaging performance is achieved by with a state of the art ultra-broad-bandwidth light source instead of superluminescent diodes. The ultimate availability of this UHR OCT technology strongly depends on the availability of ultra-broad-bandwidth light sources that are suitable for OCT applications.

\section{Acknowledgments}

The authors thank Leopold Schachinger from the Center for Biomedical Engineering and Physics, Medical University of Vienna, and Andreas Stingl, Tuan Le, and Alfred Tomasch from Femtolaser Produktions.

\section{References}

1. Drexler W, Morgner U, Kärtner FX, et al. In vivo ultrahigh resolution optical coherence tomography. Opt Lett. 1999;24:1221-1223.

2. Drexler W, Morgner U, Ghanta RK, et al. New technology for ultrahigh resolution optical coherence tomography of the retina. In: Lemij H, Schuman JS, eds. The Shape of Glaucoma. Berlin: Kugler Publications; 2000;75-104.

3. Drexler W, Morgner U, Ghanta RK, Schuman JS, Kärtner FX, Fujimoto JG. Ultrahigh resolution ophthalmologic optical coherence tomography. Nat Med. 2001;7:502-507.

4. Drexler W, Sattmann H, Hermann B. Enhanced visualization of macular pathology using ultrahigh resolution optical coherence tomography. Arch Ophthalmol. 2003;121:695-706.

5. Drexler W. Ultrahigh resolution optical coherence tomography. J Biomed Opt. 2004;9:47-74.

6. Huang D, Swanson EA, Lin CP, et al. Optical coherence tomography. Science. 1991;254:1178-1181.

7. Hee MR, Izatt JA, Swanson EA, et al. Optical coherence tomography of the human retina. Arch Ophthalmol. 1995;113:325-332.

8. Schuman JS, Puliafito CA, Fujimoto JG. Optical Coherence Tomography of Ocular Disease. Thorofare, NJ; Slack, Inc.; 2004.

9. Glosmann M, Hermann MB, Schubert C, Sattmann H, Ahnelt PK, Drexler W. Histological correlation of pig retina radial stratification with ultrahigh resolution optical coherence tomography. Invest Ophthalmol Vis Sci. 2003;44:1696-1703.

10. Anger EM, Hermann B, Schubert C, et al. Ultrahigh resolution optical coherence tomography of the monkey fovea: identification of retinal sublayers by correlation with semithin histology sections. Exp Eye Res, 2004;78:1117-1125.

11. Ko TH, Fujimoto JG, Duker JS, et al. Comparison of ultrahigh- and standard-resolution optical coherence tomography for imaging macular hole pathology and repair. Ophthalmology. 2004;111: 2033-2043.

12. Ergun E, Hermann B, Wirtitsch M, et al. Assessment of central visual function in Stargardt's disease/fundus flavimaculatus with ultrahigh-resolution optical coherence tomography. Invest $O p h$ thalmol Vis Sci. 2005;46:310-316.

13. Wollstein G, Paunescu LA, Ko TH. Ultrahigh-resolution optical coherence tomography in glaucoma. Ophthalmology. 2005;112: 229-237.

14. Rollins A, Kulkarni MD, Yazdanfar S, Ung-arunyawee R, Izatt JA. In vivo video rate optical coherence tomography. Opt Expr. 1998;5: 219-229.

15. Fercher AF, Hitzenberger CK, Kamp G, El-Zaiat SY. Measurement of intraocular distances by backscattering spectral interferometry. Opt Commun. 1995;117:43-48.

16. Glubovic B, Bouma BE, Tearney GJ, Fujimoto JG. Optical frequency-domain reflectometry using rapid wavelength tuning of a Cr4+:forsterite laser. Opt Lett. 1997;22:1704-1706.

17. Lexer F, Hitzenberger CK, Fercher AF, Kulharny M. Wavelengthtuning interferometry of intraocular distances. Appl Opt. 1997;36: $6548-6553$.

18. Häusler G, Lindner MW. Coherence radar and spectral radar: new tools for dermatological diagnosis. J Biomed Opt 1998;3:21-31.

19. Leitgeb RA, Hitzenberger CK, Fercher AF. Performance of Fourier domain vs. time domain optical coherence tomography. Optics Expr. 2003;11:889-894.

20. Choma MA, Sarunic MV, Yang C, Izatt JA. Sensitivity advantage of swept source and Fourier domain optical coherence tomography. Opt Expr. 2003;11:2183-2189.

21. De Boer JF, Cense B, Park BH, Pierce MC, Tearney GJ, Bouma BE. Improved signal-to-noise ratio in spectral-domain compared with time-domain optical coherence tomography. Opt. Lett. 2003;28: 2067-2069.

22. Wojtkowski M, Leitgeb R, Kowalczyk A, Bajraszewski T, Fercher AF. In-vivo human retinal imaging by Fourier domain optical coherence tomography. J Biomed Opt. 2002;7:457-463.

23. Yun SH, Tearney GJ, de Boer JF, Iftimia N, Bouma BE. High-speed optical frequency-domain imaging. Opt Expr. 2003;11, 22:29532963. 
24. Nassif NA, Cense B, Park BH, et al. In vivo high-resolution videorate spectral-domain optical coherence tomography of the human retina and optic nerve. Opt Expr. 2004;12:367-376.

25. Leitgeb RA, Drexler W, Unterhuber A, et al. Ultrahigh resolution Fourier domain optical coherence tomography. Opt Expr. 2004; 12, 10:2157-2165.

26. Wojtkowski M, Srinivasan VJ, Ko TH, Fujimoto JG, Kowalczyk A, Duker JS. Ultrahigh-resolution, high-speed, Fourier domain optical coherence tomography and methods for dispersion compensation. Opt Expr. 2004;12:2404-2422.

27. Cense B, Nassif NA, Chen TC, et al. Ultrahigh-resolution high-speed retinal imaging using spectral-domain optical coherence tomography. Opt Expr. 2004;12:2435-2447.

28. Leitgeb RA, Schmetterer L, Drexler W, Bajraszewski T, Zawadzki RJ, Fercher AF. Real-time assessment of retinal blood flow with ultrafast acquisition by color Doppler Fourier domain optical coherence tomography. Opt Expr. 2003;11:3116.

29. White BR, Pierce MC, Nassif $N$, et al. In vivo dynamic human retinal blood flow imaging using ultra-high-speed spectral domain optical coherence tomography. Opt Expr. 2003;11:3490.

30. Podoleanu AG, Dobre GM, Jackson DA. En-face coherence imaging using galvanometer scanner modulation. Opt Lett. 1998;23:147149 .

31. Podoleanu AG, Seeger M, Dobre GM, Webb DJ, Jackson DA, Fitzke F. Transversal and longitudinal images from the retina of the living eye using low coherence reflectometry. J Biomed Opt. 1998;3:12-20.
32. Podoleanu AG, Rogers JA, Jackson DA, Dunne S. Three-dimensional OCT images from retina and skin. Opt Exp. 2000;7:292-298.

33. Hitzenberger CK, Trost P, Lo PW, Zhou Q. Three-dimensional imaging of the human retina by high-speed optical coherence tomography. Opt Exp. 2003;11:2753-2761.

34. Podoleanu AG, Dobre GM, Cucu RC. Combined multiplanar optical coherence tomography and confocal scanning ophthalmoscopy. J Biomed Opt. 2000;9:86-93.

35. Unterhuber A, Hermann B, Sattmann H, et al. Compact, low cost Ti: $\mathrm{Al}_{2} \mathrm{O}_{3}$ laser for in vivo ultrahigh resolution optical coherence tomography. Opt Lett. 2003;28:905-907.

36. American National Standards Institute. American National Standard for Safe Use of Lasers. Washington, DC: American National Standards Institute; 2000;136.1.

37. Schmidt-Erfurth U, Teschner S, Noack J, Birngruber R. Threedimensional topographic angiography in chorioretinal neovascular disease. Invest Ophthalmol Vis Sci. 2001;42:2386-2394.

38. Haouchine B, Massin P, Tadayoni R, Erginay A, Gaudric A. Diagnosis of macular pseudoholes and lamellar macular holes by optical coherence tomography. Am J Opbthalmol. 2004;138:732-739.

39. Kusuhara S, Teraoka M, Fujii S, et al. Prediction of postoperative visual outcome based on hole configuration by optical coherence tomography in eyes with idiopathic macular holes. Am J Ophthalmol. 2004;138:709-716.

40. Chan A, Duker J, Schuman J, Fujimoto JG. Stage 0 macular holes. Opbthalmology. 2004;111:2027-2032. 